\title{
Immunotherapy With the SQ Tree SLIT-tablet in Adults and
}

\section{Adolescents With Allergic Rhinoconjunctivitis}

\section{Makela, Mika J.}

2018-04

Makela , M J , Gyllfors , P , Valovirta , E , Steffensen, M A , Gronager , P M , Savolainen , J \& Winther , L 2018 , ' Immunotherapy With the SQ Tree SLIT-tablet in Adults and Adolescents With Allergic Rhinoconjunctivitis ', Clinical Therapeutics, vol. 40 , no. 4 , pp. 574-586 . https://doi.org/10.1016/j.clinthera.2018.02.012

http://hdl.handle.net/10138/301993

https://doi.org/10.1016/j.clinthera.2018.02.012

unspecified

publishedVersion

Downloaded from Helda, University of Helsinki institutional repository.

This is an electronic reprint of the original article.

This reprint may differ from the original in pagination and typographic detail.

Please cite the original version. 


\title{
Immunotherapy With the SQ Tree SLIT-tablet in Adults and Adolescents With Allergic Rhinoconjunctivitis
}

\author{
Mika J. Mäkelä, $\mathrm{MD}^{1}$; Pär Gyllfors, MD, $\mathrm{PhD}^{2}$; Erkka Valovirta, $\mathrm{MD}^{3,4}$; \\ Maria A. Steffensen, MSc, PhD ${ }^{5}$; Pernille M. Grønager, BSc ${ }^{5}$; Johannes Savolainen, MD ${ }^{6}$; \\ and Lone Winther, $\mathrm{MD}^{7}$
}

${ }^{1}$ Skin and Allergy Hospital, Helsinki University Hospital, Helsinki, Finland; ${ }^{2}$ Asthma \& Allergy

Department, St. Göran's Hospital, Sweden; ${ }^{3}$ Department of Pulmonary Diseases and Clinical Allergology, University of Turku, Turku, Finland; ${ }^{4}$ Terveystalo Allergy Clinic, Helsinki, Finland; ${ }^{5}$ Global Research \& Development, ALK, Hørsholm, Denmark; 'Department of Pulmonary Diseases and Clinical Allergology, University of Turku, Turku, Finland; and ${ }^{7}$ Department of Dermato-Allergology, Copenhagen University Hospital, Copenhagen, Denmark

\begin{abstract}
Purpose: The SQ tree sublingual immunotherapy (SLIT)-tablet containing allergen extracts with the major allergen Bet $\mathrm{v} 1$ from birch pollen is currently being developed for the treatment of tree polleninduced allergic rhinitis/conjunctivitis with or without asthma. The aim of this Phase II trial was to investigate the dose-related efficacy and safety of the SQ tree SLIT-tablet.

Methods: This study was a randomized, parallelgroup, double-blind, placebo-controlled, multi-national trial conducted in Europe. A total of 637 participants were randomized equally to receive placebo or treatment with the SQ tree SLIT-tablet in doses of $0.5,1,2,4,7$, or 12 development units (DU). Treatment was initiated $\sim 16$ weeks before onset of the 2013 birch pollen season (BPS) and was continued throughout the BPS with a total duration of at least 6 months. During the BPS and tree pollen season (TPS), subjects assessed rhinoconjunctivitis symptoms and medication use on a daily basis in an electronic diary; weekly assessments of rhinoconjunctivitis quality of life were also made.
\end{abstract}

Findings: Analysis of the average daily symptom score during the BPS and the TPS showed that the difference between active treatment and placebo was statistically significant for the 7 DU group (BPS, $P=$ 0.02; TPS, $P=0.03$ ), with no clear dose-response relationship. All doses of the SQ tree SLIT-tablet induced changes from baseline in birch-specific $\operatorname{IgE}$ and $\mathrm{IgG}_{4}$ that were statistically significant compared with placebo at all time points assessed $(P<0.0001)$ with a clear dose-response relationship for birch specific $\mathrm{IgG}_{4}$. In general, the SQ tree SLIT-tablet was well tolerated, with the majority of treatment-related adverse events $(\geq 95 \%)$ being mild or moderate in severity. The most frequently reported treatmentrelated adverse events were generally related to the sublingual administration of the tablet (ie, they occurred in the oral cavity).

Implications: The results from this trial suggest that the SQ tree SLIT-tablet in doses up to 12 DU has a tolerability profile suitable for at-home administration. The immunomodulatory changes indicate a dose-response relationship, but clinical efficacy parameters were inconclusive, probably due to low pollen counts, emphasizing the importance of pollen exposure for the outcome of a pollen allergy immunotherapy trial. EudraCT no: 2012-000031-59. (Clin Ther. 2018;40:574-586) (c) 2018 Elsevier HS Journals, Inc. All rights reserved.

Key words: allergic rhinoconjunctivitis, birch pollen, clinical efficacy, immunology, sublingual immunotherapy tablet, tolerability.

\footnotetext{
Accepted for publication February 16, 2018.

https://doi.org/10.1016/j.clinthera.2018.02.012

0149-2918/\$ - see front matter

(C) 2018 Elsevier HS Journals, Inc. All rights reserved.
} 


\section{INTRODUCTION}

Allergic rhinitis has been identified as one of the main reasons for visits to primary care clinics, and although usually not regarded as a severe disease, it may affect quality of life, social life, school learning performance, and work productivity, especially if the patient's symptoms are moderate to severe. Furthermore, allergic rhinitis is regarded as one of the major risk factors for the development of asthma, and $>80 \%$ of patients with asthma have rhinitis, whereas $10 \%$ to $40 \%$ of people with rhinitis experience asthma. ${ }^{1,2}$

In Europe and North America, tree pollen-induced allergic rhinitis/rhinoconjunctivitis is commonly caused by allergens from the Fagales order, which includes, among others, birch, alder, hazel, and oak. They all belong to the birch homologous group and are characterized by having Bet v 1 homologous allergens with a high sequence identity, which leads to extensive cross-reactivity. Thus, patients who are sensitized to birch pollen often also experience symptoms in response to pollen from other members of the birch homologous group, which prolongs the season and extends the geographical area that can trigger allergic reactions for these patients. ${ }^{3-5}$

A large number of pollen-allergic individuals also develop allergic symptoms against certain foods such as nuts and apples. The symptoms are manifested as a condition called pollen food syndrome, and its occurrence typically involves presensitization to pollen allergens from the Fagales order. The condition is IgE-mediated and is caused by cross-reactive allergens. $^{6}$

Recommendations for the treatment of allergic diseases are allergen avoidance, symptomatic medications such as antihistamines and corticosteroids, and allergy immunotherapy (AIT). ${ }^{2}$ Avoidance of pollen can be difficult to achieve in a normal daily life, and allergy pharmacotherapy is widely used; however, it does not offer causal treatment of the allergic disease, and up to $44 \%$ of patients on optimal pharmacotherapy report poor or only partial symptom control. ${ }^{7}$

AIT is the only available treatment modality with the potential to modify the natural course of the allergic disease by induction of tolerance. ${ }^{2}$ The use of pollen extract for AIT is well known, both in subcutaneous formulations ${ }^{8-12}$ and as sublingual immunotherapy tablets or drops (SLIT-tablets/ drops). ${ }^{13,14}$ Birch pollen is recommended as a representative allergen source for AIT targeting allergies caused by pollen from the birch homologous group. This recommendation is based on results from in vitro inhibition studies in which birch pollen has shown a high degree of inhibition of human $\operatorname{IgE}$ binding to alder, hazel, and oak allergen extracts. $^{3,15,16}$

Although the exact mode of action of SLIT has not been completely established, the downstream effects include induction of $\mathrm{IgG}_{4}$ at the expense of $\operatorname{IgE}$ production. $\operatorname{IgG}_{4}$ antibodies compete with $\mathrm{IgE}$ antibodies for the binding of allergen and thus block the cross-binding of immune cell-associated IgE. ${ }^{17,18}$ Decreased allergen-specific IgE, as well as increased $\operatorname{IgG}_{1}$ and $\mathrm{IgG}_{4}$, have been shown to be hallmarks of efficacious SLIT. ${ }^{17,19}$

A tree pollen SLIT-tablet containing allergen extracts with the major allergen Bet $\mathrm{v} 1$ from birch pollen is currently being developed by ALK (Hørsholm, Denmark) for the treatment of tree pollen-induced allergic rhinitis and/or conjunctivitis. The aim of the present Phase II trial was to investigate the dose-related efficacy and safety of the SQ tree SLITtablet not only during the birch pollen season (BPS) but also during other tree pollen seasons (TPSs) such as hazel and alder.

\section{PATIENTS AND METHODS Ethics}

The trial was designed and conducted in accordance with the principles of the Declaration of Helsinki (1964, and its amendments and subsequent clarifications $)^{20}$ and was conducted in compliance with the principles of Good Clinical Practice. ${ }^{21}$ The trial was approved by the local independent ethics committes/ institutional review board and/or the health authority (as applicable) in each involved country before initiation.

\section{Trial Design}

This randomized, parallel-group, double-blind, placebo-controlled, multinational, multisite trial was conducted in Europe. The trial included 65 sites in 7 countries (Denmark, Finland, Lithuania, the Netherlands, Norway, Poland, and Sweden). A total of 637 participants were randomized equally to receive placebo or treatment with the SQ tree SLIT-tablet in doses of $0.5,1,2,4,7$, or 12 development units (DU). 


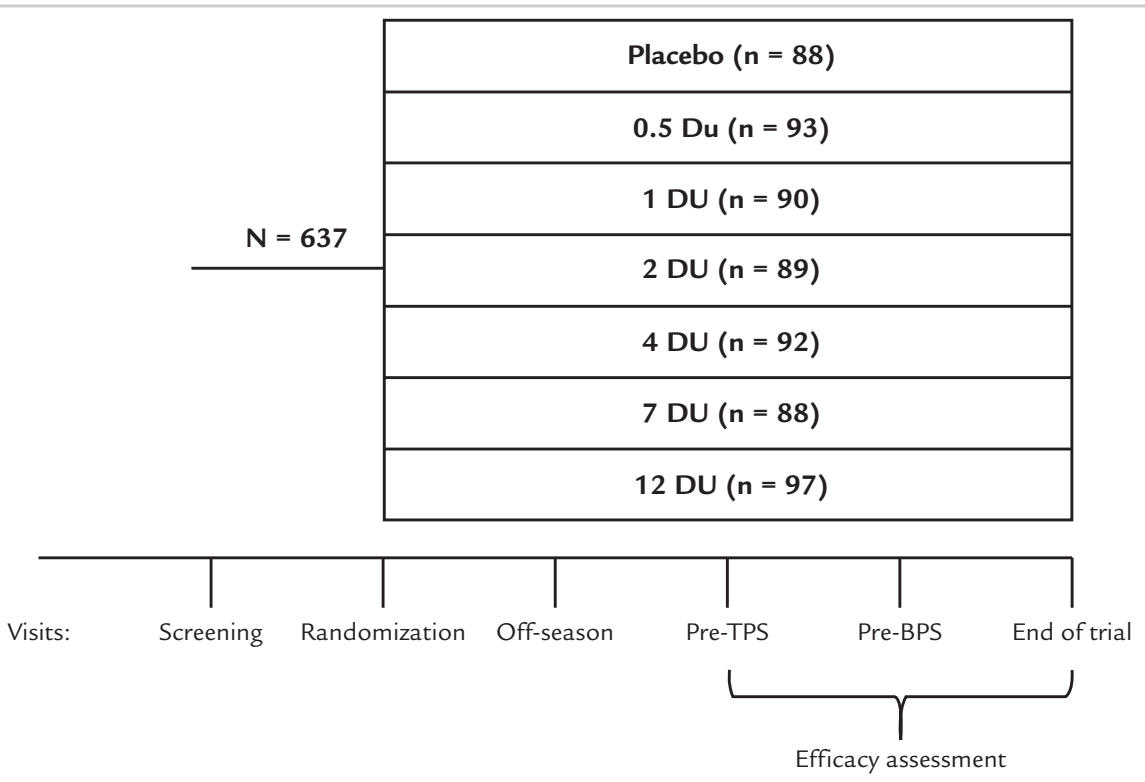

Figure 1. Trial overview. Participants were randomized equally to receive treatment with one of 6 doses of the SQ tree SLIT-tablet or placebo. Treatment was initiated $\sim 16$ weeks before onset of the 2013 birch pollen season (BPS) and was continued throughout the BPS with a total duration of at least 6 months. DU = development unit; TPS $=$ tree pollen season.

Treatment was initiated $\sim 16$ weeks before onset of the 2013 BPS and was continued throughout the BPS, with a total duration of at least 6 months. The trial had first subject first visit on August 20, 2012, and last subject last visit on July 5, 2013. The trial design, including scheduled visits and treatment doses, is illustrated in Figure 1.

\section{Trial Population}

Written informed consent was obtained at the screening visit before any other trial-related activities; for subjects aged 15 to 17 years, subject consent and parent/guardian consent was required, and for subjects aged 12 to 14 years, subject assent and parent/ guardian consent was required. The trial population consisted of adults and adolescents aged 12 to 65 years with a history of moderate to severe birch pollen-induced allergic rhinoconjunctivitis (with or without asthma), with symptoms despite having received pharmacotherapy during the 2011 and 2012 BPSs. Subjects were also required to have had an appropriate minimum level of birch pollen-induced rhinoconjunctivitis symptoms during the previous BPS (ie, 2012) defined as a daily symptom score (DSS) $\geq 8$ on the worst day of the season. Symptoms during the previous BPS were recorded by use of a retrospective questionnaire. Further selection criteria included presence of one or more of 4 Allergic Rhinitis and its Impact on Asthma quality of life items ${ }^{2}$ (sleep disturbance; impairment of daily activities, leisure and/or sport; impairment of school or work; troublesome symptoms) caused by allergic rhinoconjunctivitis during the previous BPS, positive skin prick test (SPT) response (wheal diameter $\geq 3 \mathrm{~mm}$ larger than the negative control) toward birch, positive specific IgE to Bet $\mathrm{v} 1$ (IgE class 2 or higher; $\geq 0.70 \mathrm{kU} / \mathrm{L}$ ), no clinically relevant history of perennial allergic rhinitis and/or asthma due to an allergen to which the participant is regularly exposed (eg, house dust mite, pets), no clinical history of uncontrolled asthma within 3 months before randomization, and no reduced lung function (defined as $\mathrm{FEV}_{1}<70 \%$ in adults or $\mathrm{FEV}_{1}<80 \%$ in adolescents), no treatment with immunotherapy containing birch pollen allergen within the last 5 years (or a cross-reacting allergen such as hazel or alder).

\section{Intervention Medication}

Each participant was randomly assigned to receive 1 of 6 active doses of the SQ tree SLIT-tablet or 
placebo. The first dose was administered under medical supervision lasting at least 60 minutes after tablet intake. Participants were instructed to take 1 tablet sublingually each day. The SQ tree SLIT-tablet is an oral lyophilisate containing allergen extract derived from birch pollen (Betula verrucosa), which was standardized against an in-house reference based on biological activity and defined in DUs. The intervention medication was provided and manufactured by the trial sponsor (ALK). The placebo tablet was also an oral lyophilisate and was similar to the active intervention medication with regard to appearance, smell, taste, and packaging. Thus, the placebo tablets were manufactured so that they could not be distinguished from the active intervention medication by the subject or the investigator. Standardized pharmacotherapy for rhinoconjunctivitis symptoms (ie, desloratadine tablets and olopatadine eye drops or mometasone furoate nasal spray) was provided by ALK to participants at randomization as predefined open-label medication; it was to be used freely in addition to the intervention medication to which the participants had been randomized.

\section{Randomization}

Randomization was performed according to a sponsor-generated allocation schedule by a trial-independent statistician. Randomization was stratified according to sites by using block randomization. The randomization list was divided into blocks of 7 ; that is, for each 7 numbers, there was 1 set of each dose including placebo. Allocation of randomization numbers to each site was initially done in blocks of 7 numbers. For resupplies, a complete block of 7 numbers was allocated to a site as for initial shipments. If $<7$ numbers were allocated to a site, the remaining numbers in that block were then kept free for later allocation to the same site if needed. For resupplies when complete 7-number blocks were no longer available, randomization numbers from partly used blocks were allocated. To the extent possible, partly used blocks were only allocated to sites within the same country. All subjects enrolled were identifiable throughout the trial by a 5-digit subject number starting at 50001 allocated to the subjects at the screening visit. When a subject was randomized to treatment, a 4-digit randomization number starting at 1001 was also assigned. The investigator was to always allocate the lowest randomization number available at the trial site to the subject. Randomization codes were kept strictly confidential, accessible only to authorized persons until the time of unblinding. At the end of the trial, all randomization code envelopes were collected, and reconciliation was performed between any opened code envelopes and the code breaks the sponsor had been notified about. The randomization code was not broken for any subject during the trial.

\section{End Points and Assessments}

Participants were instructed to complete symptom and medication assessments and to record the results in an electronic diary on a daily basis (in the evening before bedtime) during the TPS. Investigators notified subjects on when to start and stop entering data into the electronic diary based on information on onset and end of pollen seasons derived from the website of European Pollen Information Ltd. Efficacy assessments were based on all diary entries recorded during the 2013 TPS (discussed later).

In total, 4 rhinitis symptoms (runny nose, blocked nose, sneezing, and itchy nose) and 2 conjunctivitis symptoms (gritty feeling/red/itchy eyes and watery eyes) were measured on a scale from 0 to 3 , where 0 corresponds to no symptoms and 3 to severe symptoms. For the medication score, subjects reported their use of standardized pharmacotherapy.

The primary end point was the average rhinoconjunctivitis DSS during the BPS (range, 0-18) for the per-protocol (PP) analysis set (as discussed in the Statistical Methods section). Secondary end points included average daily (rhinoconjunctivitis) medication score (DMS) (range, 0-20) and average daily total combined score (range, 0-38) calculated as the sum of the average DSS and DMS for the full analysis set (FAS) (as discussed in the Statistical Methods section). Details of scoring scales are provided in Supplemental Tables I and II in the online version at https://doi.org/10.1016/j.clinthera.2018.02.012.

Disease-specific quality of life was assessed for adults by using the Rhinoconjunctivitis Quality of Life Questionnaire (RQLQ) with standardized activities; this questionnaire was adapted from Juniper et $\mathrm{al}^{22}$ and was included in the electronic diary on a weekly basis. Global evaluation of efficacy was performed at the end-of-trial visit at which participants were asked to compare rhinoconjunctivitis symptoms during the 2013 BPS versus the symptoms in the previous BPS 
(2012); subjects answering "better" or "much better" were categorized as "improved," and subjects answering "much worse," "worse," or "the same" were categorized as "not improved."

Immunologic parameters were assessed to confirm the diagnosis and evaluate the treatment-induced specific immune response. Blood samples were drawn for determination of allergen-specific $\operatorname{IgE}$ and $\mathrm{IgG}_{4}$ levels at the screening visit, off-season visit, pre-TPS visit, preBPS visit, and at the end-of-trial visit (Figure 1). The samples were analyzed by the research department at ALK using ImmunoCAP assays (Thermo Fisher Scientific, Waltham, Massachusetts). IgE was measured in $\mathrm{kU} / \mathrm{L}$ and $\mathrm{IgG}_{4}$ in $\mathrm{mg}_{\mathrm{A}} / \mathrm{L}$.

Safety assessments included adverse events (AEs), vital signs, physical examination, and clinical laboratory investigations. Participants received a paper notebook to complete every time they experienced an $\mathrm{AE}$, and this $\log$ served as the basis for AE assessment. AEs were recorded from when the participants signed the informed consent form and until the final follow-up; possible relation to treatment was assessed by the investigator.

\section{Definition of TPS}

Pollen counts were provided by European Pollen Information Ltd. The BPS was defined with a start date as the first day of 3 consecutive days with a birch pollen count $\geq 30$ grains $/ \mathrm{m}^{3}$ and a stop date as the last day in the last occurrence of 3 consecutive days with a birch pollen count $\geq 30$ grains $/ \mathrm{m}^{3}$. The tree pollen season was defined as the days included in any of the hazel (defined by counts $\geq 5$ grains $/ \mathrm{m}^{3}$ ), alder (defined by counts $\geq 10$ grains $/ \mathrm{m}^{3}$ ), or BPSs and was not necessarily a continuous period in time.

\section{Statistical Methods}

Results were analyzed by using SAS version 8.02 (SAS Institute, Inc, Cary, North Carolina) and R.3.1.1 (R Foundation for Statistical Computing, Vienna, Austria). All statistical analyses and CIs were 2-sided, and a significance level of $5 \%$ was used. Group comparisons regarding symptom and medication scores were analyzed by using a linear mixed effect model. RQLQ scores were analyzed in a repeated measurement model. Pollen region was included as a factor in the analysis model; that is, imbalances in means between pollen stations were reflected in the adjusted mean, resulting in an estimated mean of an average person at an average pollen station. Baseline comparability, immune responses, and safety assessments were evaluated by summary statistics and shift tables.

Two analysis sets were defined for this trial: the FAS, defined as all randomized participants (637 participants), and the PP analysis set, defined as all participants in the FAS with no major protocol deviations that affect the primary efficacy end point (453 participants). The PP set was defined by the following 3 criteria: at least $70 \%$ treatment compliance, at least $50 \%$ diary records in the BPS, and no major protocol deviations. The PP set constituted the primary dataset for the primary efficacy analysis.

Because this study was a Phase II trial, the statistical analyses were mainly exploratory, and no formal sample size calculation was performed. A sample size of 85 was chosen for each dose group because this number was expected to give a good precision of the mean of the primary end point (the average rhinoconjunctivitis DSS during the BPS). In addition, this sample size allowed for the detection of very common AEs (frequency $>10 \%$ ) with high probability $(>99 \%)$ and of common AEs ( $>1 \%$ to $\leq 10 \%$ ) with a probability of $>55 \%$ within each dose group.

\section{RESULTS}

\section{Subject Disposition and Baseline Characteristics}

Table I presents an overview of subject disposition. There were no major differences between groups in terms of numbers of discontinuations and reasons for discontinuation. However, there was a tendency toward more discontinuations due to an $\mathrm{AE}$ in the active treatment groups; $3 \%$ to $10 \%$ of participants in the active treatment groups discontinued due to an $\mathrm{AE}$ versus $3 \%$ in the placebo group. A PP analysis set was defined for each group, and the percentage of subjects included in the PP analysis set was slightly lower for the 12 DU group compared with the other treatment groups.

Table II presents the baseline characteristics. All treatment groups were similar with regard to age, sex, and years with birch pollen-induced rhinoconjunctivitis. The majority $(70 \%-81 \%)$ of participants experienced pollen food syndrome as diagnosed according to the questionnaire, whereas $25 \%$ to $35 \%$ had asthma. In accordance with the inclusion criteria, all subjects had 


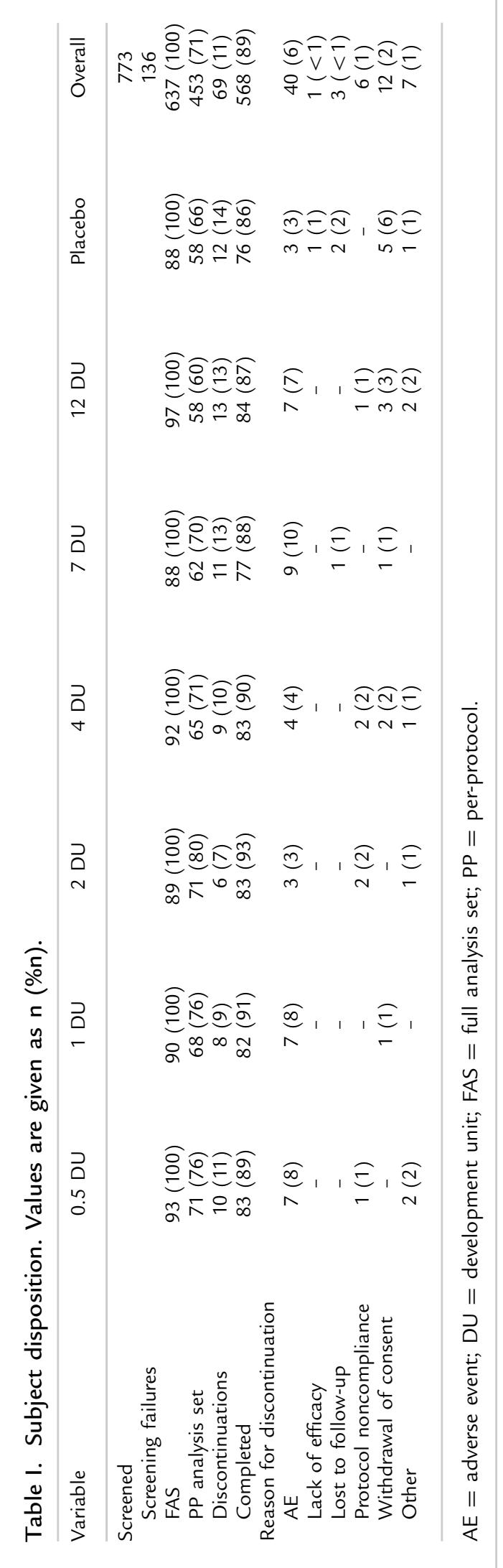

moderate to severe birch pollen-induced rhinoconjunctivitis and a positive SPT response toward birch. Furthermore, $>90 \%$ also had a positive SPT response toward alder and hazel. All treatment groups were similar in terms of rhinoconjunctivitis symptoms experienced during the $2012 \mathrm{BPS}$, as well as the extent and type of pharmacotherapy used during the 2012 BPS (data not shown).

\section{Pollen Exposure During the 2013 BPS}

The trial was conducted during a pollen season that was unusually low for birch as well as alder and hazel pollen counts. Because some pollen regions (eg, Finland) did not have birch pollen counts $>30$ grains $/ \mathrm{m}^{3}$, and thus per definition did not have a BPS (representing 147 subjects; $30 \%$ of the FAS), a BPS with limits of 10 grains $/ \mathrm{m}^{3}$ was used for all BPS results presented here. The average 2013 BPS (threshold, 30 grains $/ \mathrm{m}^{3}$ ) lasted 10 days, and the median birch pollen exposure in 2013 was 114 grains $/ \mathrm{m}^{3}$ per day for pollen regions included in the trial. This situation is considered both a short BPS and a low daily exposure level.

\section{Rhinoconjunctivitis Symptoms and Medication Scores}

The primary efficacy end point was the average DSS during the 2013 BPS, which was planned to be analyzed by fitting appropriate dose-response curves to the data from the BPS. However, as evident from Figure 2A, which shows the mean rhinoconjunctivitis DSS for the PP analysis set, the DSS did not display a dose-response relationship. Therefore, none of the planned models for fitting the data was appropriate to execute, and a linear mixed effect model was used instead.

As can be seen in Table III and Figure 2, analysis of the average DSS during the BPS for the PP analysis set found that the difference between active treatment and placebo was significant for the 7 DU group (absolute difference, $1.1 ; P=0.02$; relative difference, $33 \%$ ). Analysis of the average DSS during the TPS for the PP set also showed that the difference between active treatment and placebo was statistically significant for the 7 DU group (absolute difference, $0.8 ; P=0.03$; relative difference, $30 \%$ ) with no clear dose-response relationship. The difference between active treatment and placebo in average DSS during the BPS and the TPS was not statistically significant for the FAS (data not shown). 


\begin{tabular}{|c|c|c|c|c|c|c|c|}
\hline Variable & $\begin{array}{c}0.5 \mathrm{DU} \\
(\mathrm{n}=93)\end{array}$ & $\begin{array}{c}1 \mathrm{DU} \\
(\mathrm{n}=90)\end{array}$ & $\begin{array}{c}2 \mathrm{DU} \\
(\mathrm{n}=89)\end{array}$ & $\begin{array}{c}4 \mathrm{DU} \\
(\mathrm{n}=92)\end{array}$ & $\begin{array}{c}7 \mathrm{DU} \\
(\mathrm{n}=88)\end{array}$ & $\begin{array}{c}12 \mathrm{DU} \\
(\mathrm{n}=97)\end{array}$ & $\begin{array}{l}\text { Placebo } \\
(n=88)\end{array}$ \\
\hline \multicolumn{8}{|l|}{ Age, $y$} \\
\hline Mean (SD) & $34.7(13.1)$ & $35.4(13.3)$ & $36.2(13.5)$ & $35.5(13.4)$ & $36.6(13.5)$ & $37.5(12.7)$ & $37.6(12.6)$ \\
\hline Min-max & $12-62$ & $12-65$ & $12-65$ & $12-64$ & $12-65$ & $12-62$ & $12-64$ \\
\hline \multicolumn{8}{|l|}{ Sex, no. (\%) } \\
\hline Female & $44(47)$ & $45(50)$ & $48(54)$ & $46(50)$ & $38(43)$ & $48(49)$ & $40(45)$ \\
\hline Male & $49(53)$ & $45(50)$ & $41(46)$ & $46(50)$ & $50(57)$ & $49(51)$ & $48(55)$ \\
\hline \multicolumn{8}{|l|}{ BMI, $\mathrm{kg} / \mathrm{m}^{2}$} \\
\hline Mean (SD) & $24.6(3.7)$ & $24.8(4.0)$ & $25.2(4.8)$ & $25.5(4.8)$ & $24.8(4.1)$ & $25.7(4.9)$ & $25.3(4.6)$ \\
\hline Min-max & $18-38$ & $17-36$ & $18-45$ & $15-39$ & $18-38$ & $16-49$ & $16-45$ \\
\hline \multicolumn{8}{|c|}{ Ethnic origin, no. (\%) } \\
\hline White & $91(98)$ & $89(99)$ & $85(96)$ & $87(95)$ & $84(95)$ & $94(97)$ & $85(97)$ \\
\hline Asian & $2(2)$ & $1(1)$ & $1(1)$ & $2(2)$ & $2(2)$ & $1(1)$ & $1(1)$ \\
\hline African & - & - & $2(2)$ & $2(2)$ & - & $1(1)$ & - \\
\hline Hispanic & - & - & $1(1)$ & - & $1(1)$ & - & $1(1)$ \\
\hline Other & - & - & - & $1(1)$ & $1(1)$ & $1(1)$ & $1(1)$ \\
\hline \multicolumn{8}{|c|}{ Years with birch pollen-induced rhinoconjunctivitis, no. (\%) } \\
\hline Mean (SD) & $17.0(11.7)$ & $14.6(10.3)$ & $15.9(10.8)$ & $15.4(10.5)$ & $16.7(11.2)$ & $16.0(11.4)$ & $17.9(12.5)$ \\
\hline Min-max & $1.6-50.0$ & $0.7-42.3$ & $1.0-42.0$ & $0.6-49.4$ & $1.0-52.0$ & $1.5-43.0$ & $1.1-50.0$ \\
\hline \multicolumn{8}{|c|}{ Birch pollen-induced asthma, no. (\%) } \\
\hline Yes & $1(1)$ & $4(4)$ & $4(4)$ & $2(2)$ & $1(1)$ & $1(1)$ & $3(3)$ \\
\hline \multicolumn{8}{|c|}{ Unspecified asthma, no. (\%) } \\
\hline Yes & $29(31)$ & $25(28)$ & $28(31)$ & $27(29)$ & $21(24)$ & $34(35)$ & $23(26)$ \\
\hline \multicolumn{8}{|c|}{ Pollen food syndrome, no. (\%) } \\
\hline Yes & $65(70)$ & $70(78)$ & $66(74)$ & $69(75)$ & $71(81)$ & $70(72)$ & $67(76)$ \\
\hline
\end{tabular}

$\mathrm{BMI}=$ body mass index; $\mathrm{DU}=$ development unit; Min-max = minimum-maximum. 
A

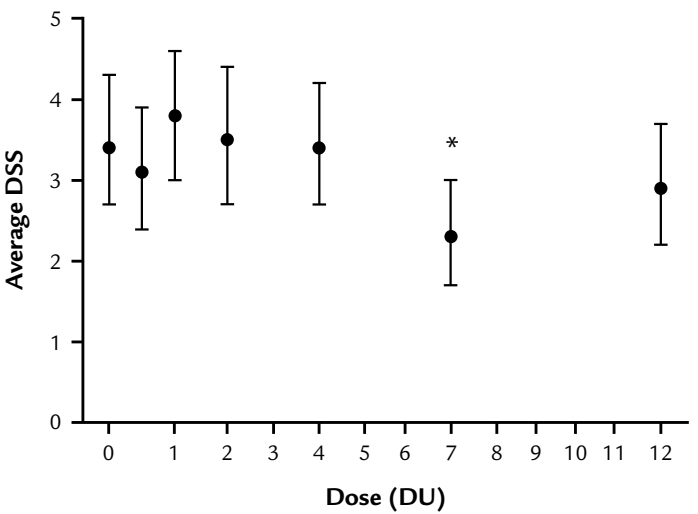

B

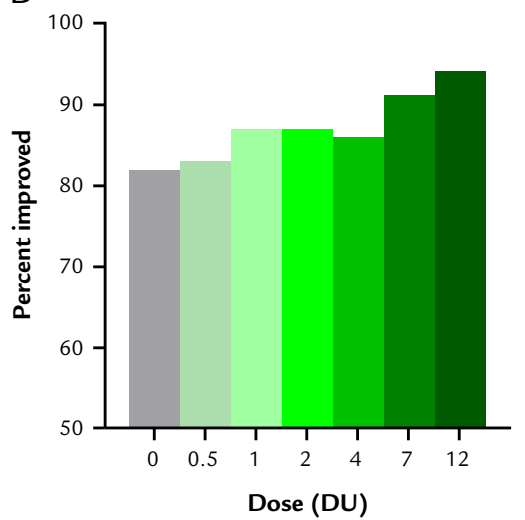

Figure 2. Clinical efficacy. (A) Average rhinoconjunctivitis daily symptom score (DSS) during the 2013 birch pollen season (BPS) for the per-protocol analysis set shown as adjusted means with $95 \% \mathrm{Cls}$. In total, 4 rhinitis symptoms (runny nose, blocked nose, sneezing, and itchy nose) and 2 conjunctivitis symptoms (gritty feeling/red/itchy eyes and watery eyes) were measured on a scale from 0 to 3 , where 0 corresponds to no symptoms and 3 to severe symptoms. (B) Global evaluation of efficacy for the full analysis set. Subjects were asked to evaluate their symptoms during the 2013 BPS compared with their symptoms during the 2012 BPS season. Subjects answering "better" or "much better" were categorized as "improved," and subjects answering "much worse," "worse," or "the same" were categorized as "not improved." DU = development unit. The asterisk indicates statistical significance.

The DMS during the BPS and the TPS was generally low for all treatment groups, and there was no statistically significant difference between active treatment and placebo for either the PP analysis set or the FAS (Table IIII and data not shown).

As observed for the rhinoconjunctivitis DSS, the total combined score for the 7 DU group during the BPS and the TPS seemed to be lower than for the rest of the groups, but there was no statistically significant difference between active treatment and placebo for either the PP analysis set or the FAS (data not shown).

Average overall RQLQ scores during both the BPS and the TPS were $\sim$, implying that subjects' quality of life were on average "hardly troubled at all" by rhinoconjunctivitis symptoms. Analysis of the average RQLQ scores showed no statistically significant difference from placebo for the PP analysis set or for the FAS (Table III and data not shown).

Global evaluation of efficacy revealed that the vast majority of participants $(>80 \%)$ had improved compared with the previous year, which was in accordance with the 2013 pollen season being unusually low. Although the primary efficacy assessment did not reveal any dose-response relationship, there seemed to be a trend toward a dose-response relationship for the global evaluation, with more participants improving in the 12 DU group (94\%) than in the placebo group (80\%) (Figure 2B). However, because the $95 \%$ CIs were overlapping, the differences are not likely to be statistically significant.

\section{Immunology}

Figure 3 illustrates the change from baseline in birch-specific $\mathrm{IgE}$ and $\mathrm{IgG}_{4}$ from the screening visit and until the end-of-trial visit for the FAS. All doses of the SQ tree SLIT-tablet induced changes from baseline in birch-specific $\operatorname{IgE}$ and $\mathrm{IgG}_{4}$ that were statistically significant compared with placebo at all time points assessed $(P<0.0001)$.

As evident from Figure 3A, IgE levels increased in all active treatment groups from the screening visit and until the off-season visit $\sim 40$ to 50 days after initiation of treatment, after which the $\operatorname{IgE}$ levels began to level off or even decrease for the 3 higher dose groups (4, 7, and $12 \mathrm{DU})$. There was no clear dose-response relationship for birch-specific IgE; however, the 3 higher doses (4, 7, and $12 \mathrm{DU})$ tended to induce a higher change from baseline than 
Table III. Analysis of daily symptom score (DSS), daily medication score (DMS), and Rhinoconjunctivitis Quality of Life Questionnaire (RQLQ) for the longer birch pollen season.

\begin{tabular}{|c|c|c|c|c|}
\hline Treatment & No. & Adjusted Means & Difference From Placebo (95\%) Cl & $P$ \\
\hline \multicolumn{5}{|l|}{ DSS (PP) } \\
\hline Placebo & 57 & 3.4 & - & - \\
\hline $0.5 \mathrm{DU}$ & 70 & 3.1 & $0.3(-0.7$ to 1.3$)$ & 0.53 \\
\hline $1 \mathrm{DU}$ & 67 & 3.8 & $-0.4(-1.3$ to 0.7$)$ & 0.51 \\
\hline $2 \mathrm{DU}$ & 71 & 3.5 & $-0.02(-1.1$ to 1.0$)$ & 0.96 \\
\hline $4 \mathrm{DU}$ & 65 & 3.4 & $0.1(-0.9$ to 1.0$)$ & 0.91 \\
\hline $7 \mathrm{DU}$ & 61 & 2.3 & $1.1(0.2$ to 2.1$)$ & 0.02 \\
\hline $12 \mathrm{DU}$ & 58 & 2.9 & $0.5(-0.5$ to 1.6$)$ & 0.30 \\
\hline \multicolumn{5}{|l|}{ DMS (PP) } \\
\hline Placebo & & 2 & - & - \\
\hline $0.5 \mathrm{DU}$ & & 1.3 & $0.7(-0.6$ to 1.9$)$ & 0.29 \\
\hline $1 \mathrm{DU}$ & & 2.4 & $-0.5(-1.9$ to 0.9$)$ & 0.5 \\
\hline $2 \mathrm{DU}$ & & 1.7 & $0.2(-1$ to 1.5$)$ & 0.7 \\
\hline $4 \mathrm{DU}$ & & 1.5 & $0.4(-0.8$ to 1.7$)$ & 0.5 \\
\hline $7 \mathrm{DU}$ & & 1.4 & $0.5(-0.7$ to 1.8$)$ & 0.4 \\
\hline $12 \mathrm{DU}$ & & 2.3 & $-0.3(-1.8$ to 1.1$)$ & 0.66 \\
\hline \multicolumn{5}{|l|}{ RQLQ (PP) } \\
\hline Placebo & & 0.8 & - & - \\
\hline $0.5 \mathrm{DU}$ & & 0.8 & $0.01(-0.3$ to 0.3$)$ & 0.9 \\
\hline $1 \mathrm{DU}$ & & 1 & $-0.1(-0.5$ to 0.2$)$ & 0.3 \\
\hline $2 \mathrm{DU}$ & & 0.9 & $-0.04(-0.3$ to 0.2$)$ & 0.8 \\
\hline $4 \mathrm{DU}$ & & 0.8 & $0.07(-0.2$ to 0.4$)$ & 0.7 \\
\hline $7 \mathrm{DU}$ & & 0.6 & $0.2(-0.05$ to 0.5$)$ & 0.1 \\
\hline $12 \mathrm{DU}$ & & 0.9 & $-0.06(-0.4$ to 0.3$)$ & 0.7 \\
\hline
\end{tabular}

the lower doses $(0.5,1$, and 2 DU). Birch-specific $\operatorname{IgE}$ remained largely unchanged in the placebo group throughout the trial at all time points assessed.

Birch-specific $\mathrm{IgG}_{4}$ increased throughout the trial in all active treatment groups with a clear dose-response relationship, whereas $\mathrm{IgG}_{4}$ remained constant at all time points assessed in the placebo group (Figure $3 \mathbf{B}$ ).

Thus, the SQ tree SLIT-tablet had a clear immune modulatory effect with the higher doses $(4,7$, and 12 DU) inducing higher changes from baseline in immunologic parameters than the lower doses $(0.5,1$, and 2 DU).

\section{Safety}

In general, the SQ tree SLIT-tablet had a favorable safety profile, with the majority of treatment-related
AEs $(\geq 95 \%)$ being of mild or moderate severity. A total of 518 participants reported a total of 2333 treatment-related AEs, and there were more subjects with treatment-related AEs in the active treatment groups compared with placebo (Supplemental Table III presents an overview of AEs in the online version at https://doi.org/10.1016/j.clinthera.2018.02. 012). The number of reported treatment-related AEs followed a dose-response trend with more treatmentrelated AEs being reported by participants in the higher dose groups. Severe treatment-related AEs were only reported by participants in the active treatment groups (68 AEs in 36 participants), but there was no clear dose-response relationship. The majority of severe treatment-related AEs were related to the sublingual administration of the tablet (ie, the 

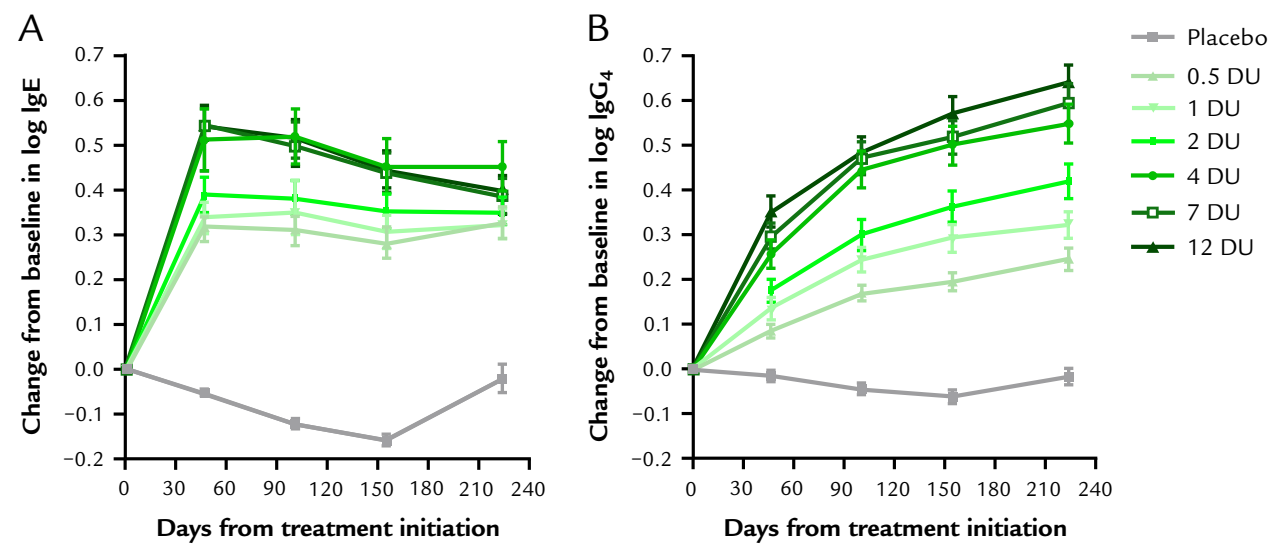

Figure 3. Immunology. Change from baseline in birch-specific (A) $\lg E$ and $(B) \lg G_{4}$ from the screening visit and until the end-of-trial visit for the full analysis set. The graphic shows means with SEM. All doses of the SQ tree sublingual immunotherapy tablet induced changes from baseline in birch-specific IgE and $\operatorname{lgG}_{4}$ that were statistically significant compared with placebo at all time points assessed. $D U=$ development unit.

AEs occurred in the oral cavity) or to the allergic disease, and they were not clustered within 1 particular system organ class or preferred term (see Supplemental Table IV in the online version at https://doi.org/ 10.1016/j.clinthera.2018.02.012). Thirty severe treatment-related AEs were treated with intervention medication belonging to the drug classes of antihistamines, over-the-counter pain-relieving medication, corticosteroids, and bronchodilators. All subjects reporting a severe treatment-related AE made

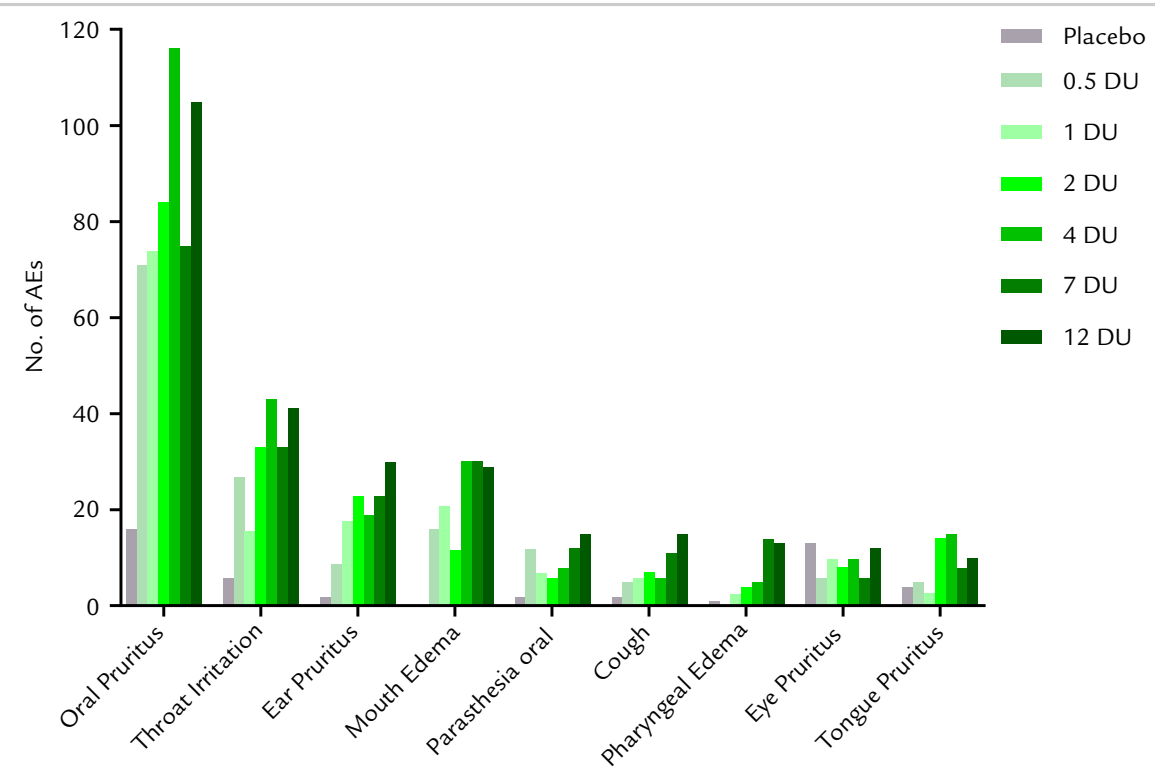

Figure 4. Most frequent treatment-related adverse events (AEs). Most frequently reported AEs were defined as those occurring in $>10 \%$ of subjects in any treatment group. The graphic shows cumulative numbers for each treatment group. DU = development unit. 
a full recovery. One treatment-related severe event of hypersensitivity was reported in the 4 DU group, but the event did not lead to discontinuation, and the subject made a full recovery. One treatment-related serious AE concerning severe asthma was reported in the 1 DU group. The participant was treated with antihistamine, corticosteroid, adrenaline, and oxygen, and the event led to discontinuation from the trial after 8 days of treatment. The subject made a full recovery.

Thirty-one participants in the active treatment groups discontinued due to 62 treatment-related AEs that were not dependent on dose. Three participants in the placebo group discontinued due to 6 treatmentrelated AEs.

The most frequently reported treatment-related AEs (occurring in $>10 \%$ of subjects in any treatment group) are illustrated in Figure 4. There was no clear dose-response relationship for any of the most frequently reported AEs, but these were reported by more participants $(81 \%[\mathrm{n}=444])$ in the active treatment groups compared with placebo $(36 \%$ [n = 32]). The majority of the most frequently reported treatment-related AEs were related to the sublingual administration of the tablet (ie, they occurred in the oral cavity). The 4 most frequently reported related AEs were oral pruritus, throat irritation, ear pruritus, and mouth edema; oral pruritus was reported most frequently. The majority of the most frequently occurring treatment-related AEs had onset within a few days after treatment initiation, resolved within $\leq 2$ months, and were mild or moderate in severity $(\geq 97 \%$ for any treatment group).

No treatment-related changes were observed with regard to clinical laboratory assessments, physical examinations, or vital signs, and there were no major differences between treatment groups in any of the parameters assessed.

\section{DISCUSSION}

This Phase II trial was designed to investigate the dose-response of the SQ tree SLIT-tablet with respect to clinical end points, immunology, and safety. Six different doses were included to give a thorough knowledge of the dose-related efficacy of the SQ tree SLIT-tablet and provide a basis for selecting the proper dose for the treatment of individuals allergic to tree pollen. The trial did not report a dose-response relationship for the primary end point (the rhinoconjunctivitis DSS) during the BPS, nor for the secondary end points DSS during the TPS and DMS and total combined score during the BPS and TPS.

The TPS in 2013 was very weak, with median pollen counts reported from pollen stations located near trial sites of 114 grains $/ \mathrm{m}^{3}$ per day. The accumulated pollen counts reported from pollen stations located near finish sites, which included 150 of the 637 trial participants, were 331 to 895 pollen grains/ $\mathrm{m}^{3}$ in 2013. For reference, an analysis of airborne birch pollen in Finland from 1974 to 2004 reported accumulated pollen counts of 590 to 38,713 grains/ $\mathrm{m}^{3},{ }^{23}$ underlining the fact that 2013 was a highly unusual year in terms of birch pollen. An earlier study showed that clinical efficacy of pollen AIT is closely associated with the level of pollen exposure, ${ }^{24}$ and this finding is in accordance with the results from our study. Generally, the clinical efficacy of SLIT has been shown to improve with increasing allergen dose, ${ }^{25,26}$ and thus, it is most likely that the 12 DU dose would show the same clinical efficacy as the 7 DU dose in a setting with higher pollen exposure; another dosefinding study is necessary to establish the optimal dose for clinical efficacy of the SQ tree SLIT-tablet. Dose-finding studies can also be performed by using allergen exposure chambers as stated in the European Medicines Agency guideline on the clinical development of products for specific immunotherapy for the treatment of allergic diseases. This approach is independent of pollen counts and is a good alternative to field trials that are highly dependent on pollen counts, which may vary greatly from year to year. Dose findings from Phase II trials performed in allergen exposure chambers should then be confirmed during a larger Phase III field trial.

In contrast to clinical efficacy, immunologic assessments are independent of pollen exposure and may thus provide a basis for evaluation of the dose-related efficacy of the SQ tree SLIT-tablet. Currently, the exact correlation between immunologic factors such as $\mathrm{IgE}$ and $\mathrm{IgG}_{4}$ and clinical efficacy as defined by reduced rhinoconjunctivitis symptoms and medications use is not known; however, immunologic assessments may provide an indicator for the immunologic response to treatment. ${ }^{27}$ This trial included 2 immunologic end points: change from baseline in $\mathrm{IgE}$ and $\mathrm{IgG}_{4}$. Both immunologic parameters showed a dose-response trend, with doses of 4 DU or higher 
inducing greater changes from baseline compared with the lower doses of 0.5 to $2 \mathrm{DU}$; however, while serum levels of $\operatorname{IgG}_{4}$ increased throughout the trial, serum levels of IgE began to level off after 2 months of treatment. A previous study on the SQ grass SLIT-tablet has shown that increased allergenspecific serum $\operatorname{IgG}_{1}$ and $\mathrm{IgG}_{4}$, and decreased serum IgE, may be indicators of clinical efficacy. ${ }^{19}$ Thus, the immunomodulatory effects observed in this trial suggest that doses of $4 \mathrm{DU}$ or higher may provide the best treatment effect.

The safety end points are also considered pollen independent, as the majority of AEs reported with SLIT-tablets are reported at treatment initiation and therefore outside the pollen season. Generally, the SQ tree SLIT-tablet was well tolerated, with $>95 \%$ of treatment-related AEs being of mild or moderate severity. The number of treatment-related AEs followed a dose-response trend, but number of severe related AEs did not. Furthermore, the proportion of subjects who discontinued due to treatment-related AEs was similar between active treatment groups, and all doses were therefore considered tolerable and safe for at-home administration, provided that the first tablet is administered under medical supervision. The most frequently reported related AEs were local reactions related to the sublingual administration of the tablet such as oral pruritus, throat irritation, ear pruritus, and mouth edema. Onset was typically within a few days of treatment initiation, and resolution was within 2 months. The safety profile of the SQ tree SLIT-tablet trial reported here confirms previous results from a Phase I trial with the SQ tree SLIT-tablet. ${ }^{28}$ Furthermore, clinical trials conducted with the corresponding SQ grass SLIT-tablet and the SQ house dust mite SLIT-tablet showed a similar safety profile, with the majority of related AEs being local transient AEs, primarily mild or moderate in severity affecting the mouth and throat. ${ }^{29,30}$

\section{CONCLUSIONS}

The results from this trial suggest that the SQ tree SLIT-tablet in doses up to $12 \mathrm{DU}$ has a tolerability profile suitable for at-home administration. The immunomodulatory changes indicated a dose-response relationship, but clinical efficacy parameters were inconclusive, probably due to low pollen counts, emphasizing the importance of pollen exposure for the outcome of a pollen AIT trial. Another Phase II dose-finding trial assessing symptoms during controlled allergen exposure in an allergen exposure chamber was initiated after completion of the TT-02 trial.

\section{ACKNOWLEDGMENTS}

The authors thank trial statistician Jens Strodl Andersen and the ALK trial team for their excellent work. Drs. Mäkelä, Gyllfors, Savolainen, and Winther were responsible for the study design, execution of the study, and interpretation of results; Grønager was responsible for the immunologic analyses and interpretation of results; and Dr. Steffensen was responsible for the interpretation of results and article preparation. All authors reviewed and approved the final article.

The trial was sponsored by ALK.

\section{CONFLICTS OF INTEREST}

Drs. Steffensen and Grønager are employed by ALK. The trial was sponsored by ALK, which was involved in study design; collection, analysis and interpretation of data; writing of the manuscript; and the decision to submit the manuscript for publication. The authors have indicated that they have no other conflicts of interest regarding the content of this article.

\section{SUPPLEMENTARY MATERIAL}

Supplemental tables accompanying this article can be found in the online version at https://doi:10.1016/j. clinthera.2018.02.012.

\section{REFERENCES}

1. Bousquet J, Van Cauwenberge P, Khaltaev N, Aria Workshop Group, World Health Organization. Allergic rhinitis and its impact on asthma. J Allergy Clin Immunol. 2001;108:S147-S334.

2. Bousquet J, Khaltaev N, Cruz AA, et al. Allergic Rhinitis and its Impact on Asthma (ARIA) 2008 update (in collaboration with the World Health Organization, GA(2)LEN and AllerGen). Allergy. 2008;63(Suppl 86):8-160.

3. Lorenz AR, Luttkopf D, May S, et al. The principle of homologous groups in regulatory affairs of allergen productsa proposal. Int Arch Allergy Immunol. 2008;148:1-17.

4. Asam C, Hofer H, Wolf M, et al. Tree pollen allergens-an update from a molecular perspective. Allergy. 2015;70: 1201-1211. 
5. Mothes N, Horak F, Valenta R. Transition from a botanical to a molecular classification in tree pollen allergy: implications for diagnosis and therapy. Int Arch Allergy Immunol. 2004;135:357-373.

6. Katelaris $\mathrm{CH}$. Food allergy and oral allergy or pollen-food syndrome. Curr Opin Allergy Clin Immunol. 2010;10:246-251.

7. Valovirta E, Myrseth SE, Palkonen S. The voice of the patients: allergic rhinitis is not a trivial disease. Curr Opin Allergy Clin Immunol. 2008;8:1-9.

8. Bousquet J, Lockey R, Malling HJ. Allergen immunotherapy: therapeutic vaccines for allergic diseases. A WHO position paper. J Allergy Clin Immunol. 1998;102:558-562.

9. Lang R, Hawranek T. Hymenoptera venom immunotherapy and field stings. J Investig Allergol Clin Immunol. 2006;16:224-231.

10. Blumberga G, Groes L, Dahl R. SQstandardised house dust mite immunotherapy as an immunomodulatory treatment in asthma patients. Allergy. 2011;66:178-185.

11. Winther L, Malling $\mathrm{HJ}$, Mosbech $\mathrm{H}$. Allergen-specific immunotherapy in birch- and grass-pollen-allergic rhinitis. II. Side-effects. Allergy. 2000;55:827-835

12. Winther L, Malling HJ, Moseholm L, Mosbech H. Allergen-specific immunotherapy in birch- and grass-pollen-allergic rhinitis. I. Efficacy estimated by a model reducing the bias of annual differences in pollen counts. Allergy. 2000;55:818-826.

13. Demoly P, Emminger W, Rehm D, et al. Effective treatment of house dust mite-induced allergic rhinitis with 2 doses of the SQ HDM SLITtablet: results from a randomized double-blind, placebo-controlled phase III trial. J Allergy Clin Immunol. 2016; 137:444-451.

14. Durham SR, Emminger W, Kapp A, et al. SQ-standardized sublingual grass immunotherapy: confirmation of disease modification 2 years after 3 years of treatment in a randomized trial. J Allergy Clin Immunol. 2012;129:717-725.

15. Niederberger $V$, Pauli G, Gronlund $\mathrm{H}$, et al. Recombinant birch pollen allergens (rBet $\vee 1$ and rBet $\vee 2$ ) contain most of the IgE epitopes present in birch, alder, hornbeam, hazel, and oak pollen: a quantitative IgE inhibition study with sera from different populations. J Allergy Clin Immunol. 1998;102:579-591.

16. Hauser M, Asam C, Himly M, et al. Bet $v$ 1-like pollen allergens of multiple Fagales species can sensitize atopic individuals. Clin Exp Allergy. 2011;41:1804-1814.

17. Jay DC, Nadeau KC. Immune mechanisms of sublingual immunotherapy. Curr Allergy Asthma Rep. 2014;14: 473.

18. Fujita H, Soyka MB, Akdis M, Akdis CA. Mechanisms of allergen-specific immunotherapy. Clin Transl Allergy. 2012;2:2.

19. Suarez-Fueyo A, Ramos T, Galan A, et al. Grass tablet sublingual immunotherapy downregulates the $\mathrm{TH} 2$ cytokine response followed by regulatory T-cell generation. J Allergy Clin Immunol. 2014;133:130-138.

20. World Medical Association. Declaration of Helsinki: ethical principles for medical research involving human subjects. 2008. Adopted by the WMA General Assembly in Helsinki (1964) and as amended by the WMA General Assembly.

21. $\mathrm{ICH}$. ICH Harmonised Tripartite Guideline Topic E6 (R1): Guideline for Good Clinical Practice. 1996.

22. Juniper EF, Thompson AK, Ferrie PJ, Roberts JN. Validation of the standardized version of the Rhinoconjunctivitis Quality of Life Questionnaire. J Allergy Clin Immunol. 1999;104:364-369.
23. Yli-Panula E, Fekedulegn DB, Green BJ, Ranta H. Analysis of airborne betula pollen in Finland; a 31-year perspective. Int J Environ Res Public Health. 2009;6:1706-1723.

24. Durham SR, Nelson HS, Nolte H, et al. Magnitude of efficacy measurements in grass allergy immunotherapy trials is highly dependent on pollen exposure. Allergy. 2014;69:617-623.

25. Calderon MA, Larenas D, KleineTebbe J, et al. European Academy of Allergy and Clinical Immunology task force report on 'dose-response relationship in allergen-specific immunotherapy'. Allergy. 2011;66: 1345-1359.

26. Demoly P, Passalacqua G, Calderon MA, Yalaoui T. Choosing the optimal dose in sublingual immunotherapy: rationale for the 300 index of reactivity dose. Clin Transl Allergy. 2015;5:44.

27. Matsuoka T, Shamji MH, Durham SR. Allergen immunotherapy and tolerance. Allergol Int. 2013;62: 403-413.

28. Birk AO, Andersen JS, Villesen $\mathrm{HH}$, et al. Tolerability of the SQ Tree SCIT tablet in adults. Clin Ther. 2017;39:1858-1867.

29. Durham SR, Yang WH, Pedersen $M R$, et al. Sublingual immunotherapy with once-daily grass allergen tablets: a randomized controlled trial in seasonal allergic rhinoconjunctivitis. J Allergy Clin Immunol. 2006;117:802-809.

30. Mosbech H, Deckelmann R, de Blay $F$, et al. Standardized quality (SQ) house dust mite sublingual immunotherapy tablet (ALK) reduces inhaled corticosteroid use while maintaining asthma control: a randomized, double-blind, placebocontrolled trial. J Allergy Clin Immunol. 2014;134:568-575.
Address correspondence to: Maria Abildgaard Steffensen, MSc, PhD, ALK, Bøge Allé 1, 2970 Hørsholm, DK-Denmark. E-mail: mafdk@alk.net 


\section{SUPPLEMENTARY MATERIAL}

Supplementary Tables I-IV.

Supplementary Table I. Sympton scoring scale.

Rhinoconjunctivitis symptoms Daily score

Rhinitis symptoms

Runny nose $\quad 0-3$

Blocked nose $\quad 0-3$

Sneezing 0-3

Itchy nose $\quad 0-3$

Rhinitis DSS 0-12

Conjunctivitis symptoms

Gritty feeling/red/itchy eyes 0-3

Watery eyes $\quad 0-3$

Conjunctivitis DSS 0-6

Rhinoconjunctivitis DSS 0-18

Supplementary Table II. Pharmacotherapy scoring scale.

\begin{tabular}{lcc}
\hline Rhinoconjunctivitis pharmacotherapy & Score/dose unit & Daily score \\
\hline Desloratadine tablets $(5 \mathrm{mg} /$ tablet $)$ & 6 & $0-6$ \\
Olopatine eye drops $(1 \mathrm{mg} / \mathrm{ml})$ & 1.5 & $0-6$ \\
Mometasone furoate nasal spray $(50 \mathrm{pg} /$ puff) & 1 & $0-8$ \\
Rhinoconjunctivitis DMS & & $0-20$
\end{tabular}




\begin{tabular}{|c|c|c|c|c|c|c|c|c|c|c|c|c|c|c|}
\hline & \multicolumn{2}{|c|}{$0.5 \mathrm{DU}$} & \multicolumn{2}{|c|}{$1 \mathrm{DU}$} & \multicolumn{2}{|c|}{$2 \mathrm{DU}$} & \multicolumn{2}{|c|}{$4 \mathrm{DU}$} & \multicolumn{2}{|c|}{$7 \mathrm{DU}$} & \multicolumn{2}{|c|}{$12 \mathrm{DU}$} & \multicolumn{2}{|c|}{ Placebo } \\
\hline & \multicolumn{2}{|c|}{$N=93$} & \multicolumn{2}{|c|}{$N=90$} & \multicolumn{2}{|c|}{$N=89$} & \multicolumn{2}{|c|}{$N=92$} & \multicolumn{2}{|c|}{$N=88$} & \multicolumn{2}{|c|}{$N=97$} & \multicolumn{2}{|c|}{$N=88$} \\
\hline & $\begin{array}{c}n \\
(\% n)\end{array}$ & $\begin{array}{c}\mathrm{e} \\
(\% \mathrm{e})\end{array}$ & $\begin{array}{c}n \\
(\% n)\end{array}$ & $\begin{array}{c}\mathrm{e} \\
(\% \mathrm{e})\end{array}$ & $\begin{array}{c}n \\
(\% n)\end{array}$ & $\begin{array}{c}\mathrm{e} \\
(\% \mathrm{e})\end{array}$ & $\begin{array}{c}n \\
(\% n)\end{array}$ & $\begin{array}{c}\mathrm{e} \\
(\% \mathrm{e})\end{array}$ & $\begin{array}{c}n \\
(\% n)\end{array}$ & $\begin{array}{c}\mathrm{e} \\
(\% \mathrm{e})\end{array}$ & $\begin{array}{c}n \\
(\% n)\end{array}$ & $\begin{array}{c}\mathrm{e} \\
(\% \mathrm{e})\end{array}$ & $\begin{array}{c}n \\
(\% n)\end{array}$ & $\begin{array}{c}\mathrm{e} \\
(\% \mathrm{e})\end{array}$ \\
\hline All AEs & $85(91)$ & $442(100)$ & $78(37)$ & $397(100)$ & S3 (93) & $539(100)$ & $86(93)$ & $572(100)$ & $82(93)$ & $513(100)$ & $89(92)$ & $652(100)$ & $71(31)$ & $305(100)$ \\
\hline \multicolumn{15}{|l|}{ Causality } \\
\hline $\begin{array}{l}\text { Unlikely } \\
\text { Possible }\end{array}$ & $74(80)$ & $270(61)$ & $74(82)$ & $264(64)$ & $75(84)$ & $373(69)$ & $83(90)$ & $418(73)$ & $79(90)$ & $393(77)$ & $\begin{array}{l}58(60) \\
85(88)\end{array}$ & $477(73)$ & $\begin{array}{l}57(65) \\
48(55)\end{array}$ & $\begin{array}{l}167(55) \\
138(45)\end{array}$ \\
\hline \multicolumn{15}{|l|}{ Severity } \\
\hline Mild & $80(86)$ & $304(89)$ & $73(81)$ & $277(70)$ & $78(88)$ & $422(78)$ & $80(87)$ & $425(74)$ & $77(88)$ & $346(67)$ & $80(82)$ & $447(69)$ & $59(67)$ & $207(68)$ \\
\hline Moderate & $43(46)$ & $123(28)$ & $42(47)$ & $104(26)$ & $40(45)$ & $104(19)$ & $48(52)$ & $126(22)$ & $45(51)$ & $145(28)$ & $55(57)$ & $177(27)$ & $38(43)$ & $86(28)$ \\
\hline Severe & $8(9)$ & $15(3)$ & $10(11)$ & $16(4)$ & $8(9)$ & $12(2)$ & $11(12)$ & $21(4)$ & $11(13)$ & $22(4)$ & $15(15)$ & $28(4)$ & $6(7)$ & $12(4)$ \\
\hline Unknown & - & - & - & - & $1(1)$ & $1(<1)$ & - & - & - & - & - & - & - & - \\
\hline \multicolumn{15}{|l|}{ Seriousness } \\
\hline Serious & - & - & $1(1)$ & $1(1)$ & $2(2)$ & $2(<1)$ & $1(1)$ & $1(<1)$ & - & - & $5(5)$ & $5(<1)$ & - & - \\
\hline Non-serious & $85(91)$ & $442(100)$ & $78(47)$ & $396(>99)$ & $83(93)$ & $537(>99)$ & $86(93)$ & $571(>99)$ & $82(93)$ & $513(100)$ & $89(92)$ & $647(>99)$ & $71(81)$ & $305(100)$ \\
\hline \multicolumn{15}{|l|}{ Outcome } \\
\hline Recovered & 85 (91\%) & $420(95 \%)$ & $78(87 \%)$ & 385 (97\%) & $83(93 \%)$ & $523(97 \%)$ & $85(\mathrm{~S} 2 \%)$ & $547(96 \%)$ & $82(93 \%)$ & 497 (97\%) & 89 (92\%) & 627 (96\%) & $69(78 \%)$ & $288(94 \%)$ \\
\hline Recovered with sequelae & & & & & & & & & & & $1(1 \%)$ & $1(<1 \%)$ & & \\
\hline Not recovered & $13(14 \%)$ & $18(4 \%)$ & $10(11 \%)$ & $11(3 \%)$ & $11(12 \%)$ & $15(3 \%)$ & $13(14 \%)$ & $21(4 \%)$ & $11(13 \%)$ & $13(3 \%)$ & $13(13 \%)$ & $23(4 \%)$ & $7(8 \%)$ & $10(3 \%)$ \\
\hline Unknown & $4(4 \%)$ & $4(<1 \%)$ & $1(1 \%)$ & $1(<1 \%)$ & $1(1 \%)$ & $1(<1 \%)$ & $2(2 \%)$ & $4(<1 \%)$ & $3(3 \%)$ & $3(<1 \%)$ & $1(1 \%)$ & $1(<1 \%)$ & $3(3 \%)$ & $7(2 \%)$ \\
\hline $\begin{array}{l}\text { AES leading to } \\
\text { discontinuation }\end{array}$ & $7(8)$ & $14(3)$ & $7(8)$ & $14(4)$ & $3(3)$ & $4(<1)$ & $4(4)$ & $15(3)$ & $9(10)$ & $13(3)$ & $7(7)$ & $16(2)$ & $3(3)$ & $6(2)$ \\
\hline
\end{tabular}




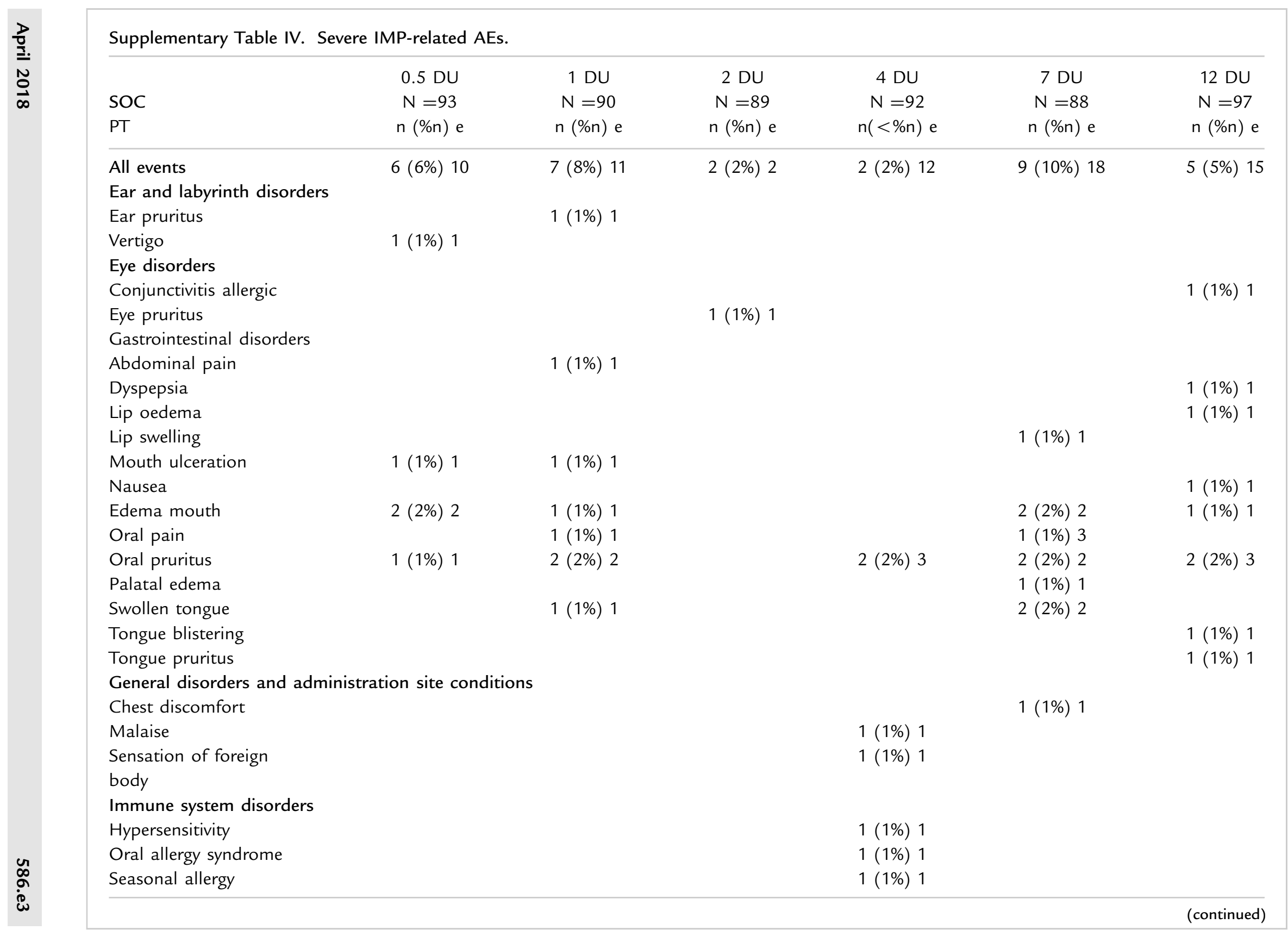




\begin{tabular}{|c|c|c|c|c|c|c|}
\hline \multicolumn{7}{|c|}{ Supplementary Table IV. (continued). } \\
\hline & $0.5 \mathrm{DU}$ & $1 \mathrm{DU}$ & $2 \mathrm{DU}$ & $4 \mathrm{DU}$ & $7 \mathrm{DU}$ & $12 \mathrm{DU}$ \\
\hline SOC & $N=93$ & $\mathrm{~N}=90$ & $N=89$ & $\mathrm{~N}=92$ & $\mathrm{~N}=88$ & $\mathrm{~N}=97$ \\
\hline PT & $n(\% n)$ e & $n(\% n)$ e & $n(\% n)$ e & $\mathrm{n}(<\% \mathrm{n}) \mathrm{e}$ & $n(\% n)$ e & $n(\% n) e$ \\
\hline \multicolumn{7}{|c|}{ Infections and infestations } \\
\hline Nasopharyngitis & $1(1 \%) 1$ & & & & & \\
\hline \multicolumn{7}{|c|}{ Nervous system disorders } \\
\hline Aphonia & & & & $1(1 \%) 1$ & & \\
\hline Headache & & & & $1(1 \%) 1$ & & \\
\hline \multicolumn{7}{|c|}{ Respiratory, thoracic and mediastinal disorders } \\
\hline Asthma & & $1(1 \%) 1$ & & & & \\
\hline Cough & $1(1 \%) 1$ & $1(1 \%) 1$ & & $1(1 \%) 1$ & & $2(2 \%) 2$ \\
\hline Dyspnea & & & & & $2(2 \%) 2$ & \\
\hline Nasal congestion & $1(1 \%) 1$ & & & & & \\
\hline Pharyngeal edema & & & $1(1 \%) 1$ & & $2(2 \%) 2$ & \\
\hline Respiratory stress & & $1(1 \%) 1$ & & & & \\
\hline Rhinorrhea & & & & & & $1(1 \%) 1$ \\
\hline Sneezing & $1(1 \%) 1$ & & & & & \\
\hline Throat irritation & & & & & & $2(2 \%) 2$ \\
\hline Throat tightness & & & & $1(1 \%) 1$ & $1(1 \%) 1$ & \\
\hline \multicolumn{7}{|c|}{ Skin and subcutaneous tissue disorders } \\
\hline Pruritus & $1(1 \%) 1$ & & & & & \\
\hline Urticaria & & & & & $1(1 \%) 1$ & \\
\hline
\end{tabular}

